\title{
Short Communication: Neuroprotective Effect of Spirulina in a Mouse Model of ALS
}

\author{
Svitlana Garbuzova-Davis ${ }^{*}, 1-4$ and Paula C. Bickford ${ }^{1-3,5}$ \\ ${ }^{I}$ Center of Excellence for Aging and Brain Repair, University of South Florida, College of Medicine, Tampa, FL 33612, \\ USA \\ ${ }^{2}$ Department of Neurosurgery and Brain Repair, University of South Florida, College of Medicine, Tampa, FL 33612, \\ USA \\ ${ }^{3}$ Department of Molecular Pharmacology and Physiology, University of South Florida, College of Medicine, Tampa, FL \\ 33612, USA \\ ${ }^{4}$ Department of Pathology and Cell Biology, University of South Florida, College of Medicine, Tampa, FL 33612, USA \\ ${ }^{5}$ James A. Haley Veterans Affairs, Medical Center, Tampa, FL 33612, USA
}

\begin{abstract}
Nutritional approaches to the treatment of aging and neurodegenerative diseases have been shown to have potential benefits. Fruits or vegetables provide a cocktail of phytochemicals with multiple actions. Spirulina, a blue green alga used for thousands of years as a food source by the Aztecs, is known to contain large amounts of $\beta$-carotene and several phycocyanins with potent antioxidant and anti-inflammatory effects. We examined neuroprotective effects of a spirulina $0.1 \%$ supplemented diet in the G93A SOD1 mouse model of ALS beginning at 5 weeks of age and continuing for 10 weeks. Spirulina dietary supplement significantly maintained body weight and extension reflex, and reduced inflammatory markers and motor neuron degeneration in G93A mice. These findings provide initial evidence that nutrition supplementation with spirulina has a neuroprotective support for dying motor neurons. A spirulina supplemented diet may be a potential alternative or adjunctive treatment for ALS.
\end{abstract}

Keywords: G93A SOD1 mice, diet, spirulina, neuroprotection.

\section{INTRODUCTION}

Amyotrophic lateral sclerosis (ALS) is a degenerative motor neuron disease that clinically manifests as progressive muscular weakness leading to paralysis and death within three to five years of diagnosis. Most available treatments for this disease are palliative, unable to arrest disease progression or repair motor neurons. The sporadic ALS (SALS) form dominates; with only $5-10 \%$ of ALS cases being familial (FALS) or genetically linked. Clinical presentation and underlying pathology of SALS and FALS are similar.

Evidence for oxidative stress has been found in both forms of ALS, tempting one to consider the possibility that similar mechanisms are at play in disease pathogenesis $[1$, 2]. Moreover, since missense mutations in SOD1, the main free radical scavenging enzyme that protects cells against oxidative stress, have been found in FALS [3, 4], oxidative damage may play a crucial role in ALS. One theory is that mutations in SOD1 lead to a decreased affinity for $\mathrm{Zn}$ and this tips the body's cellular balance towards apoptosis and that peroxynitrite is an intermediate [5]. Peroxynitrite is a strong oxidizing agent that has been suggested as a common

*Address correspondence to this author at the Center of Excellence for Aging and Brain Repair, Department of Neurosurgery and Brain Repair, University of South Florida, College of Medicine, 12901 Bruce B. Downs Blvd., Tampa, FL 33612, USA; Tel: 813-974-3189; Fax: 813-974-3078; E-mail: sgarbuzo@health.usf.edu link in many neurodegenerative diseases and is also an important mediator of inflammation [6]. In the brainstem and spinal cord of both ALS patients and animal models, increased numbers of activated microglia and astrocytes accompanied by $\mathrm{IgG}$ deposits and infiltration of $\mathrm{T}$ lymphocytes were observed [7-10]. The increases of macrophage-secreted cytokines such as interleukin (IL) $1 \alpha$, IL $1 \beta$ and IL 1RA [11], the pro-inflammatory enzyme cyclooxygenase type 2 (Cox-2) $[8,12,13]$, and up-regulation of the tumor necrosis factor-alpha (TNF- $\alpha)$ gene $[11,14]$ make them likely candidates for the effectors damaging motor neurons. These cytokine increases preceded bulk changes in protein oxidation and apoptosis, suggesting that the inflammatory reaction is a primary event precipitating oxidation and apoptosis of motor neurons.

Our studies and others have shown the potential beneficial actions of nutritional approaches to the treatment of aging and neurodegenerative diseases [15-19]. Fruits or vegetables provide a cocktail of phytochemicals with multiple actions including antioxidant and anti-inflammatory effects. For example, blueberries are known to contain numerous phenolic compounds such as anthocyanins that are potent antioxidants; the phenolic content varies in different berry varieties [20]. Adding Gingko biloba extract (EGb761), which has antioxidant properties as a free radical scavenger, to the diet of G93A SOD1 mice significantly improved motor performance and mouse survival [21]. Spirulina, a blue green alga used for thousands of years as a 
food source by the Aztecs, is known to contain large amounts of $\beta$-carotene [22] and several phycocyanins [23], all with potent antioxidant effects. Phycocyanin is also known to have potent COX-2 inhibitory actions [24, 25], actions that may be important as COX-2 is increased in ALS [13]. Foods containing phycocyanin may have dual benefits. We have shown potent decreases in markers of oxidative damage and inflammation in aged rats fed diets supplemented with spirulina or spinach $[15,26]$. Moreover, spirulina, blueberries, and spinach can reduce ischemic damage following middle cerebral artery occlusion in rats [19], suggesting that nutritional approaches may provide a broad spectrum of neuroprotective actions in a model of neurodegeneration. In the present study, we examined neuroprotective effects of a spirulina supplemented diet in the G93A SOD1 mouse model of ALS.

Transgenic male mice B6SJL-TgN (SOD1-G93A) 1GUR (G93A; obtained from Jackson Laboratories, Bar Harbor, MA, USA) over-expressing human SOD1, carrying the Gly93 $\rightarrow$ Ala mutation, were used. Fifteen G93A mice randomly assigned to one of two groups received a regular control (NIH 31) diet $(n=7)$ or a diet supplemented with spirulina $0.1 \%(n=8)$ beginning at 5 weeks of age and continuing for 10 weeks. The control NIH 31 diet was a typical basal diet. The spirulina diet consisted of the basal diet supplemented with $0.1 \%$ spirulina powder. Mice were housed individually in cages. Food (diets) and water were available ad libitum. All described procedures were approved by the Institutional Animal Care and Use Committee at USF and conducted in compliance with the Guide for the Care and Use of Laboratory Animals. Data are presented as means \pm S.E.M and were analyzed by Mann-Whitney test. GraphPad InStat (GraphPad Software, Inc.) software was used.

\section{CHARACTERISTICS OF DISEASE PROGRESSION}

Evaluation of animal disease progression was as previously described [27-29]. All measures of disease progression were performed blind by independent investigators to avoid subjective bias. At 5 weeks of age (before beginning the diets) and then weekly until 15 weeks of age, body weights of mice were measured. Results demonstrate that G93A mice receiving the spirulina $0.1 \%$ dietary supplement significantly maintained body weights compared to G93A mice with control diet (Fig. 1A). Significant differences

\section{$\mathbf{A}$}

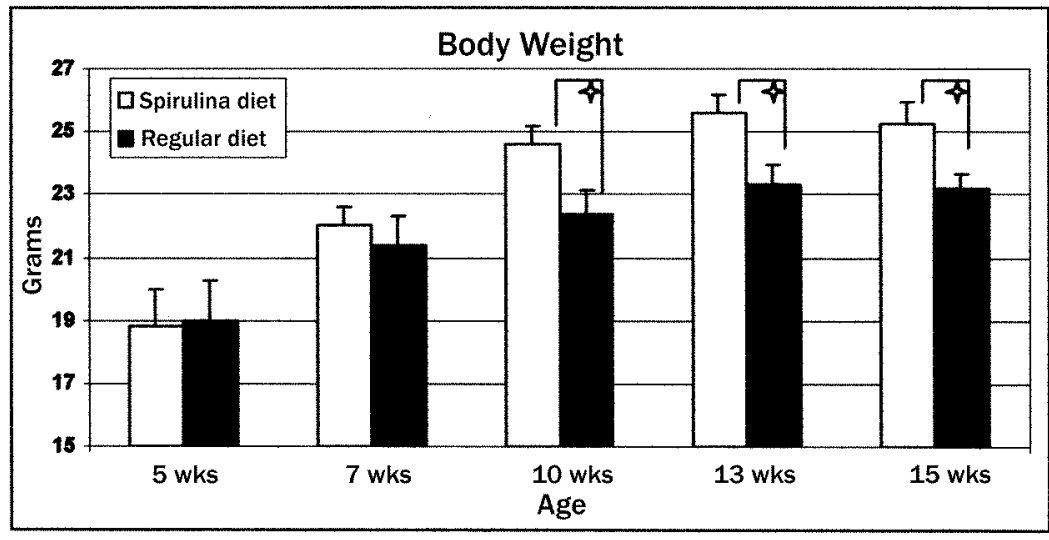

B

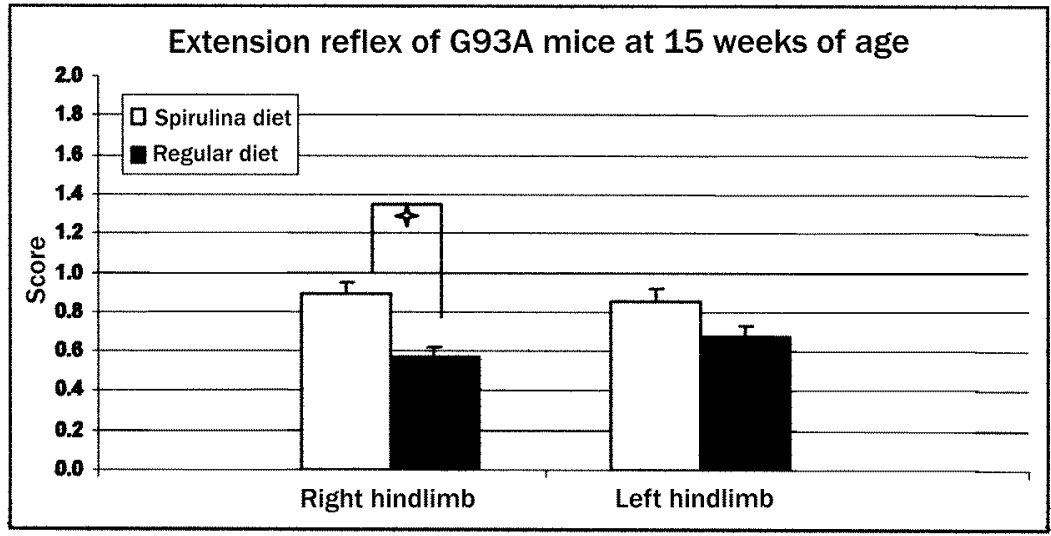

Fig. (1). Body weight and extension reflex of G93A mice with spirulina dietary supplementation. (A) G93A mice receiving the spirulina $0.1 \%$ dietary supplement starting at 5 weeks of age and continuing for the 10 following weeks significantly maintained body weights compared to G93A mice on the control diet. Significant differences $(p<0.05)$ in body weights between mice fed with spirulina or control diets were observed beginning at 10 weeks of age. (B) Extension reflex of hindlimbs of G93A mice measured at 15 weeks of age showed a significant $(p<0.05)$ effect of spirulina diet. Mice receiving spirulina for 10 weeks retained extension reflex of right hindlimbs $v s$. mice on the control diet. $p<0.05$. 
$(p<0.05)$ in body weights between mice fed with spirulina or control diets were observed beginning at 10 weeks of age, 5 weeks after beginning supplementation. At 15 weeks of age, body weights of G93A mice on the control diet were $23.2 \pm 0.44 \mathrm{~g} v s .25 .3 \pm 0.69 \mathrm{~g}$ for mice on spirulina supplementation. Although a control mice group was not included in this study, data from our previous published work [29, 30], showed that control mice such as transgenic mice (BL6/SJL) carrying the normal allele for SOD1 gene typically maintained their body weights of $\sim 30 \mathrm{~g}$ at this age. G93A mice carrying the mutant SOD1 gene usually begin experiencing decreased body weight at 13 weeks of age, when first disease symptoms appear, due to muscle atrophy. Extension reflex of each hindlimb was determined at 15 weeks of age, prior to euthanatasia, when each mouse was suspended by the tail. If the mouse showed normal hindlimb extension, a score of 2 was given. A score of 1 indicated partial hindlimb extension. If no extension was observed, the score was 0 . Mice receiving spirulina for 10 weeks retained extension reflex $v s$. mice on the regular diet (Fig. 1B). Interestingly, extension of right hindlimbs in mice fed the spirulina diet was significantly $(\mathrm{p}<0.05)$ higher vs. control diet mice. Although, extension of left highlimbs of these mice tended to be sustained, no significant differences were found between the two groups of mice. Numerous reports [31-33], including ours [27-30], have shown that disease symptoms become apparent at approximately 90 days of age (13 weeks of age) and motor deficits have been detected as early as 8 weeks of age [34] in G93A mice. In our study, G93A mice fed with spirulina for 10 weeks showed significant differences in maintaining body weight and
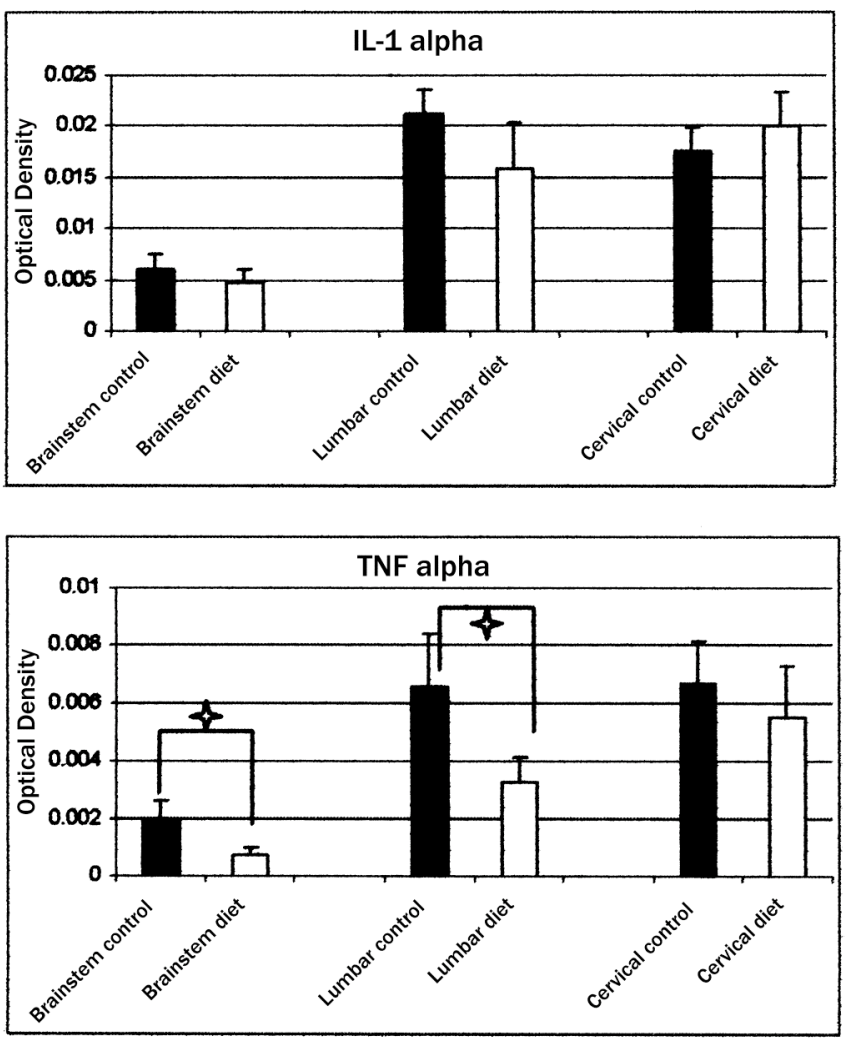

extension reflex at 15 weeks of age vs. mice on the control diet. Similar results were obtained when oral administration of green Gingko biloba extract (EGb761) was added to the diet of G93A SOD1 mice [21], significantly improving motor performance and survival of these mice.

\section{RIBONUCLEASE PROTECTION ASSAY}

Mice were sacrificed under deep pentobarbital (100 $\mathrm{mg} / \mathrm{kg}$, ip) anesthesia at 15 weeks of age. The brainstem, cervical and lumbar spinal cord segments were removed from five mice in each group, frozen in liquid nitrogen, and stored at $-80^{\circ} \mathrm{C}$. Ribonuclease Protection Assay was performed to determine cytokine profiles in the brainstem and spinal cord of mice as previously described [15]. Total RNA from homogenized tissues was extracted using Qiagen Rneasy minikit (Qiagen, Valencia, CA) according to the manufacturer's instructions. Briefly, twenty $\mu \mathrm{g}$ of total RNA from each sample was hybridized with antisense, radiolabeled probes, after which the free probe and remaining single-stranded RNA were digested with RNase A/T1. The probe (Pharmingen) included mouse specific sequences for interleukin-1 $\alpha$ (IL-1 $\alpha)$, IL-1 $\beta$ IL-6, and tumor necrosis factor $\alpha(\operatorname{TNF} \alpha)$. The phosphorimaging screen was subsequently scanned with a phosphorimager (Molecular Image System GS-363; Bio-Rad). The images were processed using Molecular Analyst software (Bio-Rad). The intensity of a band in the computer generated image was directly proportional to the amount of radioactivity within the band. The optical density (OD) values obtained from each band were normalized against the OD obtained from the L32 band
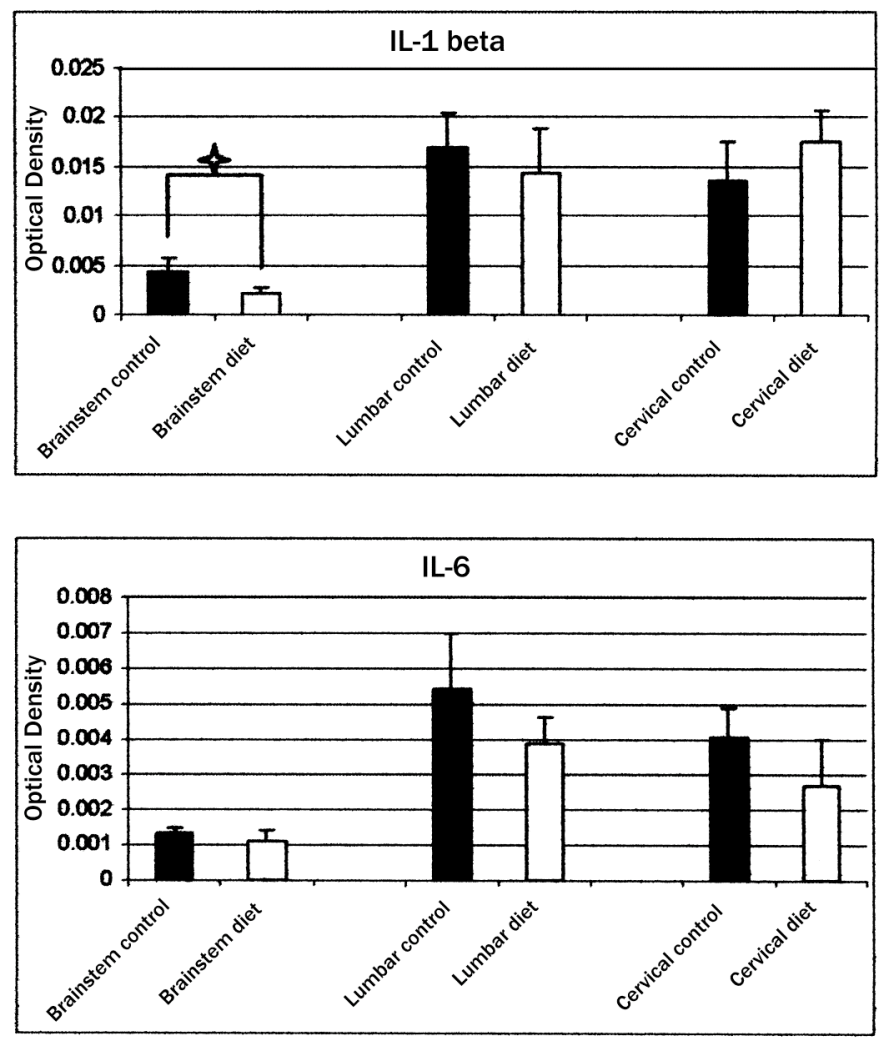

Fig. (2). Characteristics of pro-inflammatory cytokine expression in the brainstem and spinal cords of spirulina fed and control diet G93A mice at 15 weeks of age. Spirulina diet significantly $(\mathrm{p}<0.05)$ decreased IL-1 beta in the brainstem and TNF alpha in the brain and lumbar spinal cord. $p<0.05$. 
in that sample by the following expression: OD of the sample band / OD of the L32 band X 100. Significant $(p<0.05)$ decreases of IL-1 beta (brainstem) and TNF alpha (brainstem and lumbar spinal cord) were detected in spirulina-diet animals vs. control mice (Fig. 2). In the cervical spinal cord, there were no significant differences for studied cytokines between mice fed with spirulina or regular diet, however, a tendency towards decreasing TNF alpha and IL-6 in mice receiving spirulina was noted.

\section{IMMUNOHISTOCHEMISTRY}

For immunohistochemical studies, the remaining mice were perfused transcardially with $4 \%$ paraformaldehyde (PFA) in $0.1 \mathrm{M}$ phosphate buffer ( $\mathrm{PB}, \mathrm{pH} 7.2)$. The lumbar segments of the spinal cord were removed, post-fixed, and then cryoprotected in $20 \%$ sucrose in $0.1 \mathrm{M}$ PB overnight. Coronal sections $(30 \mu \mathrm{m})$ were cut in a cryostat, thawmounted on slides, and stored at $-20^{\circ} \mathrm{C}$. The lumbar spinal cords were double stained for Fluoro-Jade, a marker of degenerating neurons, and glial fibrillary acidic protein (GFAP), an astrocyte marker. The $0.001 \%$ Fluoro-Jade solution was used according to the manufacturer's protocol. After staining, the sections were incubated in a blocking solution and then polyclonal rabbit anti-GFAP antibody (1:500, Dako) was applied and the slides were incubated overnight at $4^{\circ} \mathrm{C}$. The slides were rinsed and incubated with goat-antirabbit secondary antibody (1:700, Alexa Fluor 594) conjugated to rhodamine. After several rinses, the slides were coverslipped with Vectashield (Vector Laboratories) and examined under an epifluorescence Olympus BX60 research microscope. In controls, the primary antibody was omitted. Double immunohistochemical staining for GFAP and Fluoro-Jade of the lumbar spinal cords demonstrated numerous activated astrocytes and neural degeneration in control fed mice at 15 weeks of age (Fig. 3a). Large degenerated motor neurons were detected in these mice (Fig. 3b). In G93A mice fed with spirulina supplement, consistent quailtative decreases of activated astrocytes and neural degeneration in the lumbar spinal cord were noted at the same age (Fig. 3c). Some motor neurons were partially positive for Fluoro-Jade indicating the process of degeneration (Fig. 3d).

In summary, our study results present initial evidence that nutrition supplementation with spirulina may provide neuroprotective support for dying motor neurons in a mouse model of ALS. Our results showed that the spirulina dietary
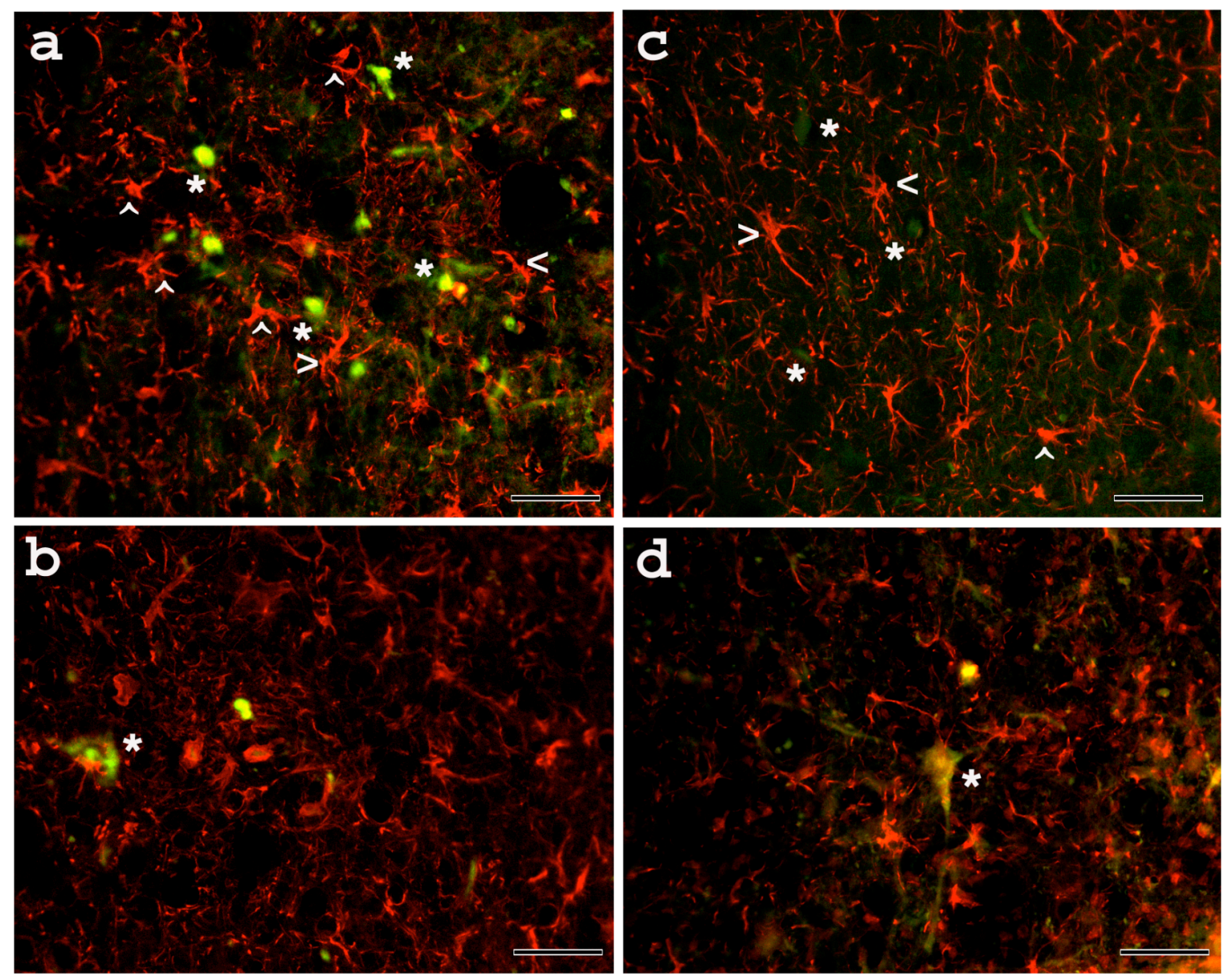

Fig. (3). Immunohistochemical staining for astrocytes (GFAP) and neural degeneration (Fluoro-Jade) in the lumbar spinal cord of G93A mice at 15 weeks of age. (a) Astrocyte activation (red, arrowheads) and neuronal degeneration (green, asterisks) in ventral horn of the lumbar spinal cord were observed in control diet G93A mice. (b) Degenerated large motor neurons (green, asterisk) were seen in control fed mice at 15 weeks of age. (c) Fewer activated astrocytes (red, arrowheads) and degenerated (green, asterisks) neurons were found in spirulina fed mice. (d) In these mice, some large motor neurons were beginning to degenerate (yellow, asterisk). Scale bar in a-d is $50 \mu \mathrm{m}$. 
supplement slowed the onset of motor symptoms and disease progression, reducing inflammatory markers and motor neuron death in G93A mice. Indications were that a reduction of inflammatory processes for preventing motor neuron degeneration might be achieved with the spirulina diet. Green Gingko biloba is considered to have protective effects against mitochondrial damage and oxidative stress [21], yet spirulina may have dual, antioxidant and anti-inflammatory, effects on motor neurons. Although motor neuron counts were not performed in this study, the quantitative analysis of motor neurons survival is important. This question and others will be addressed in our future experiments. Investigation of different concentrations of spirulina dietary supplement will be necessary to find the most beneficial nutritional approach. Also, determining the effect of a spirulina supplemented diet on lifespan of G93A mice is essential to prove the efficacy of our proposed treatment. Thus, a spirulina supplemented diet may have future clinical benefit in treating ALS as an alternative or adjunctive therapy.

\section{ACKNOWLEDGEMENTS}

This work was supported in part by the Department of Neurosurgery and Brain Repair, University of South Florida, and James A. Haley Veterans Affairs Medical Center, Tampa, Florida. We would like to acknowledge Elise Spencer's work performing cytokine assays and immunohistochemistry.

\section{CONFLICT OF INTEREST}

SGD and PCB are consultants of Saneron CCEL Therapeutics, Inc. PCB is a co-founder of Natura Therapeutics, Inc.

\section{REFERENCES}

[1] Cookson MR, Shaw PJ. Oxidative stress and motor neurone disease. Brain Pathol 1999; 9: 165-86.

[2] Robberecht W. Oxidative stress in amyotrophic lateral sclerosis. J Neurol 2000; 247(Suppl 1): 1-6.

[3] Rosen DR, Siddique T, Patterson D, et al. Mutations in $\mathrm{Cu} / \mathrm{Zn}$ superoxide dismutase gene are associated with familial amyotrophic lateral sclerosis. Nature 1993; 362: 59-62.

[4] Fiszman ML, Borodinsky LN, Ricart KC, Sanz OP, Sica RE. $\mathrm{Cu} / \mathrm{Zn}$ superoxide dismutase activity at different ages in sporadic amyotrophic lateral sclerosis. J Neurol Sci 1999; 162: 34-7.

[5] Beckman JS, Estevez AG, Crow JP, Barbeito L. Superoxide dismutase and the death of motoneurons in ALS. Trends Neurosci 2001; 24: 15-20.

[6] Torreilles F, Salman-Tabcheh S, Guerin M, Torreilles J. Neurodegenerative disorders: the role of peroxynitrite. Brain Res Rev 1999; 30: 153-63.

[7] Alexianu ME, Kozovska M, Appel SH. Immune reactivity in a mouse model of familial ALS correlates with disease progression. Neurology 2001; 57: 1282-9.

[8] McGeer PL, McGeer EG. Inflammatory processes in amyotrophic lateral sclerosis. Muscle Nerve 2002; 26: 459-70.

[9] Hall ED, Oostveen JA, Gurney ME. Relationship of microglial and astrocytic activation to disease onset and progression in a transgenic model of familial ALS. Glia 1998; 23: 249-56.

[10] Elliott JL. Cytokine upregulation in a murine model of familial amyotrophic lateral sclerosis. Brain Res Mol Brain Res 2001; 95: 172-8.

[11] Hensley K, Floyd RA, Gordon B, et al. Temporal patterns of cytokine and apoptosis-related gene expression in spinal cords of the G93A-SOD1 mouse model of amyotrophic lateral sclerosis. J Neurochem 2002; 82: 365-74.
[12] Almer G, Guegan C, Teismann P, et al. Increased expression of the pro-inflammatory enzyme cyclooxygenase- 2 in amyotrophic lateral sclerosis. Ann Neurol 2001; 49: 176-85.

[13] Maihofner C, Probst-Cousin S, Bergmann M, Neuhuber W, Neundorfer B, Heuss D. Expression and localization of cyclooxygenase-1 and -2 in human sporadic amyotrophic lateral sclerosis. Eur J Neurosci 2003; 18: 1527-34.

[14] Yoshihara T, Ishigaki S, Yamamoto M, et al. Differential expression of inflammation- and apoptosis-related genes in spinal cords of a mutant SOD1 transgenic mouse model of familial amyotrophic lateral sclerosis. J Neurochem 2002; 80: 158-67.

[15] Gemma C, Mesches MH, Sepesi B, Choo K, Holmes DB, Bickford PC. Diets enriched in foods with high antioxidant activity reverse age-induced decreases in cerebellar beta-adrenergic function and increases in proinflammatory cytokines. J Neurosci 2002; 22: 6114-20.

[16] Joseph J, Denisova N, Fisher D, Bickford P, Prior R, Cao G. Agerelated neurodegeneration and oxidative stress: putative nutritional intervention. [Review]. Neurol Clin 1998; 16: 747-55.

[17] Bickford P, Shukitt-Hale B, Joseph J. Effects of aging on cerebellar noradrenergic function and motor learning: nutritional interventions. Mech Ageing Dev 1999; 111: 141-54.

[18] Wang Y, Chang CF, Chou J, et al. Dietary supplementation with blueberries, spinach, or spirulina reduced ischemic brain damage. Exp Neurol 2005; 193: 75-84.

[19] Yasuhara T, Hara K, Maki M, et al. Dietary supplementation exerts neuroprotective effects in ischemic stroke model. Rejuvenation Res 2008; 11: 201-14.

[20] Zheng W, Wang SY. Oxygen radical absorbing capacity of phenolics in blueberries, cranberries, chokeberries, and lingonberries. J Agric Food Chem 2003; 51: 502-9.

[21] Ferrante RJ, Klein AM, Dedeoglu A, Beal MF. Therapeutic efficacy of EGb761 (Gingko biloba extract) in a transgenic mouse model of amyotrophic lateral sclerosis. J Mol Neurosci 2001; 17 : 89-96.

[22] Annapurna V, Deosthale YG, Bamji MS. Spirulina as a source of vitamin A. Plant Foods Hum Nutr 1991; 41: 125-34.

[23] Bhat V, Madyastha KM. Scavenging of peroxynitrite by phycocyanin and phycocyanobilin from Spirulina platensis: protection against oxidative damage to DNA. Biochem Biophys Res Commun 2001; 285: 262-6.

[24] Reddy C, Bhat VB, Kiranmai G, Reddy MN, Reddanna P, Madyastha KM. Selective inhibition of cyclooxygenase-2 by Cphycocyanin, a biliprotein from Spirulina platensis. Biochem Biophys Res Commun 2000; 277: 599-603.

[25] Reddy M, Subhashini J, Mahipal SV, et al. C-Phycocyanin, a selective cyclooxygenase-2 inhibitor, induces apoptosis in lipopolysaccharide-stimulated RAW 264.7 macrophages. Biochem Biophys Res Commun 2003; 304: 385-92.

[26] Cartford M, Gemma C, Bickford PC. Eighteen-month-old Fischer 344 rats fed a spinach-enriched diet show improved delay classical eyeblink conditioning and reduced expression of tumor necrosis factor alpha (TNFalpha) and TNFbeta in the cerebellum. J Neurosci 2002; 22: 5813-6.

[27] Garbuzova-Davis S, Willing AE, Milliken M, et al. Intraspinal implantation of hNT neurons into SOD1 mice with apparent motor deficit. Amyotroph Lateral Scler Other Motor Neuron Disord 2001; 2: $175-80$.

[28] Garbuzova-Davis S, Willing AE, Milliken M, et al. Positive effect of transplantation of hNT neurons (NTera 2/D1 cell-line) in a model of familial amyotrophic lateral sclerosis. Exp Neurol 2002; 174: $169-80$.

[29] Garbuzova-Davis S, Willing AE, Zigova $\mathrm{T}$, et al. Intravenous administration of human umbilical cord blood cells in a mouse model of amyotrophic lateral sclerosis: distribution, migration, and differentiation. J Hematother Stem Cell Res 2003; 12: 255-70.

[30] Willing AE, Garbuzova-Davis S, Saporta S, Milliken M, Cahill DW, Sanberg PR. hNT neurons delay onset of motor deficits in a model of amyotrophic lateral sclerosis. Brain Res Bull 2001; 56: 525-30.

[31] Gurney ME, Pu H, Chiu AY, et al. Motor neuron degeneration in mice that express a human $\mathrm{Cu}, \mathrm{Zn}$ superoxide dismutase mutation. Science 1994; 264: 1772-5.

[32] Chiu AY, Zhai P, Dal Canto MC, et al. Age dependent penetrance of disease in a transgenic mouse model of familial amyotrophic lateral sclerosis. Mol Cell Neurosci 1995; 6: 349-62. 
[33] Weydt P, Hong SY, Kliot M, Moller T. Assessing disease onset and progression in the SOD1 mouse model of ALS. NeuroReport 2003; 14: 1051-4.
[34] Barne'oud P, Lolivier J, Sanger DJ, Scatton B, Moser P. Quantitative motor assessment in FALS mice: a longitudinal study. NeuroReport 1997; 8: 2861-5.

(C) Garbuzova-Davis and Bickford; Licensee Bentham Open.

This is an open access article licensed under the terms of the Creative Commons Attribution Non-Commercial License (http: //creativecommons.org/licenses/by$\mathrm{nc} / 3.0 /$ ), which permits unrestricted, non-commercial use, distribution and reproduction in any medium, provided the work is properly cited. 